\title{
LA INVESTIGACIÓN APLICADA EN LA CUALIFICACIÓN DE LA PRÁCTICA DOCENTE
}

\author{
María Helena Quijano Hernández \\ Magíster en Educación \\ Docente de la Universidad Industrial de Santander \\ Escuela de Educación Facultad de Ciencias Humanas. \\ Bucaramanga - Colombia \\ mquijano@uis.edu.co
}

\section{Cómo citar este artículo}

Quijano, M. E. (2013) La investigación aplicada en la cualificación de la práctica docente. Espiral, Revista de Docencia e Investigación. 3 (2)

\section{Resumen}

Los cambios que marcan el transcurso del siglo XXI, conllevan la determinación de la calidad como un referente de posicionamiento global, regional y local; haciendo eco a este paradigma, los sistemas educativos consideran las pruebas censales un medio que diagnóstica la calidad de la educación, pero también constituyen un recurso comparativo entre mejores sistemas educativos, instituciones educativas, sector público y privado, contextos urbanos y rurales ¿De qué depende la calidad de los mejores sistemas educativos?, un primer factor, es la base cultural de la familia y la formación docente, como factor determinante. Una formación docente consolidada en la investigación a realizar desde el ámbito de desempeño, el aula de clase. En correspondencia con lo anteriormente planteado, el artículo inicialmente remite a los mejores sistemas educativos según los resultados de PISA, cuáles son los criterios de selección a la carrera docente; enseguida considera el documento de la UNESCO (2012) Antecedentes y criterios para la elaboración de políticas docentes en América Latina y el Caribe para mostrar algunas características de la formación docente y aspectos críticos; continua en el contexto nacional tomando como fuente el documento del MEN (2012) Políticas y sistema colombiano de formación y desarrollo profesional docente. Una segunda parte, plantea la investigación como una de las vías en la formación y calidad de los docentes; cierra el artículo con algunas experiencias en los programas de formación docente ofertados por la Escuela de Educación de la Universidad Industrial de Santander.

Palabras clave: calidad educativa, formación docente, investigación aplicada, investigación acción, práctica docente.

\footnotetext{
Abstract

The changes mark the course of the 21st century, leading to determine the quality as a bellwether for global positioning, regional and local echoing this, education systems consider evidence that diagnostic census half
}

the quality of education, but also provide a comparative resource best education systems, educational institutions, public and private sectors, urban and rural contexts. What determines the quality of the best education systems? , The first factor is the cultural foundation of the family and teacher training as a determining factor. A consolidated teacher training to conduct research from the field of performance the classroom. In correspondence with the above stated, the article initially refers to the best education systems according to the PISA results, what are the selection criterion to the teaching profession, at once considered the UNESCO document (2012) " Background and criterion for the development educational politics in Latin America and the Caribbean " to show some features of teacher education and critical aspects in the national context continues taking as the source document MEN (2012) "Politics and Colombian system of training and professional development ." A second part presents the research as one of the pathways in the formation and quality of teachers, closes the article with some experiences in teacher education programs offered by the School of Education at the Universidad Industrial de Santander.

Keywords: quality education, Teacher training, applied research, action research, teaching practice.

\section{Introducción}

La educación en la actualidad se valora en términos de calidad, dispuesta según pruebas censales nacionales como: Saber, aplicada a estudiantes de la educación básica primaria y secundaria, evalúa competencias comunicativas, científicas, matemáticas y ciudadanas; Saber 11, la presentan los estudiantes de grado once de la educación media; Saber Pro, prueba presentada por los estudiantes de educación superior al finalizar las diferentes carreras profesio- 
nales. Entre las pruebas internacionales y en las que ha participado Colombia, tenemos: Estudio Internacional de Tendencias en Matemáticas y Ciencias (TIMSS) y el Programa para la Evaluación Internacional de Alumnos (PISA), propuesto por la Organización para la Cooperación y el Desarrollo Económico (OCDE), en esta prueba participan cerca de 70 países entre miembros y no miembros de la OCDE. La finalidad según PISA, es evaluar las competencias lectoras, científicas y matemáticas. Los resultados se han utilizado para indagar por qué son mejores algunos sistemas educativos y buscan mantenerse en los primeros lugares desde que se aplican las pruebas, año 2000, es el caso de: Shanghai - China, Corea, Finlandia, Hong Kong, - China, Singapur, Canadá, Nueva Zelanda, Singapur, Australia, Holanda. El informe de PISA (2009) señala como "sistemas escolares exitosos aquellos con niveles de desempeño por encima del promedio y que muestran inequidades socioeconómicas por debajo del promedio, ofrecen a todos los estudiantes, independientemente de su condición socioeconómica, oportunidades similares para aprender" (p.20); ahora bien, Barber \& Mourshed (2008), se cuestionan cómo hicieron los sistemas educativos con mejor desempeño del mundo para alcanzar sus objetivos, y encuentran tres factores: conseguir a las personas más aptas para ejercer la docencia; formarlas hasta convertirlas en instructores eficientes; y garantizar que el sistema sea capaz de brindar la mejor instrucción posible a todos los escolares.

Considerando el primer factor, en este artículo se presentan cuál es el proceso de selección en la carrera docente, tomando como ejemplo el caso de Singapur y el de Finlandia. En el caso de América Latina y el Caribe, se exponen algunas situaciones de acuerdo con el documento de la UNESCO (2012) Antecedentes y criterios para la elaboración de políticas docentes en América Latina y el Caribe. A nivel nacional y tomando como fuente el docu- mento del MEN (2012) Políticas y sistema colombiano de formación y desarrollo profesional docente, se exponen los propósitos de la formación docente según Plan Decenal. Se aborda la investigación aplicada, como una vía en el mejoramiento y cualificación de la práctica.

\section{La calidad de un sistema educativo y la for- mación de los docentes}

Las condiciones de selección de quienes ingresan a programas profesionales de formación docente, así como las exigencias que implica el sostenerse en estos programas, constituyen parte de los aspectos que se manifiestan en la calidad de la educación. Los procesos de selección de Singapur y Finlandia, acentúan en los logros académicos de los postulantes, sus habilidades de comunicación y su motivación hacia la docencia, señalan Barber, M., \& Mourshed, M. (2008); ¿qué incluye el proceso de selección en estos sistemas?

Los mejores sistemas, seleccionan a los aspirantes antes de que den inicio a la capacitación docente, limitando los cupos del programa de capacitación a los seleccionados. Según información de video de Tonny Wagner (2011), miembro del programa de innovación educativa del centro de tecnología del emprendimiento de Harvard, el sistema educativo de Finlandia está basado en la confianza, privilegia el aprendizaje de un currículo integrado, son los estudiantes de alto nivel quienes ingresan a la profesión docente y en el transcurso de la carrera reciben formación práctica, los docentes poseen título de maestría, son profesionales y realización investigación sobre la propia práctica.

A nivel de América Latina, y de acuerdo con el documento de la UNESCO (2012):

Antecedentes y criterios para la elaboración de políticas docentes en América Latina y el Caribe, la formación docente inicia en las Escuelas Normales, consideradas instituciones de educación secundaria, cuyo propósito 
es la formación de maestros de educación primaria y, en algunos casos, de educación preescolar. Usualmente son dependientes, académica y administrativamente, de los
Ministerios o Secretarías de Educación. (p.40)

La tabla 1 y 2 muestran los aspectos y criterios de selección:

Tabla 1. Cómo hicieron los sistemas educativos con mejor desempeño del mundo para alcanzar sus objetivos. Barber, M., \& Mourshed, M. 2008.

SINGAPUR: SELECCIÓN DE DOCENTES

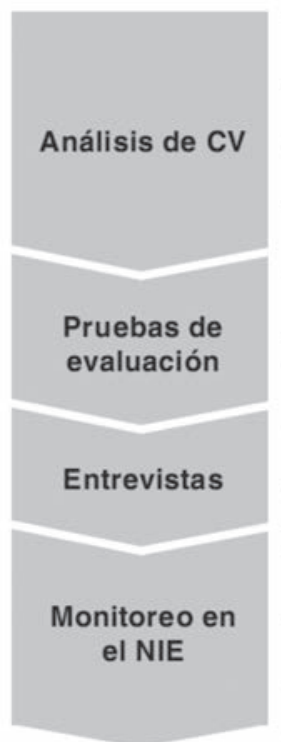

- Verificación de calificaciones mínimas:

- Académicamente, los postulantes deben estar dentro del $30 \%$ superior de su promoción.

- Los postulantes deben haber completado la educación escolar y universitaria pertinente.

- Los postulantes deben demostrar interés en los niños y en la educación.

\section{- Verificación de conocimientos.}

- Los postulantes deben tener un alto nivel de conocimientos.

- Está demostrado que los conocimientos de los docentes inciden sobre los logros más que cualquier otra variable.

- Verificación de actitud, aptitud y personalidad:

- Llevada a cabo por un panel de tres directores expertos. Puede incluir pruebas y actividades prácticas.

- Verificación de actitud, aptitud y personalidad:

- Los docentes son monitoreados durante su capacitación inicial en el NIE (Instituto Nacional de Evaluación).

- Un pequeño número de candidatos que no satisface los estándares requeridos es eliminado del curso.

Fuente: Entrevistas: Ministerio de Educación (Singapur)

Tabla 2. Cómo hicieron los sistemas educativos con mejor desempeño del mundo para alcanzar sus objetivos. Barber, M., \& Mourshed, M. 2008.

FINLANDIA: SELECCIÓN DE DOCENTES

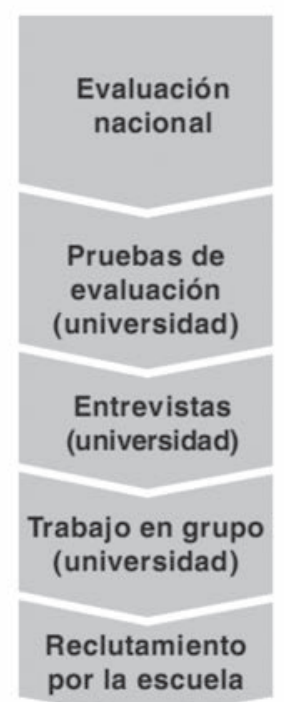

- Verificación de fuertes características intrínsecas: Evaluación tipo multiple choice de 300 preguntas sobre aritmética, lengua y resolución de problemas (desde 2007; antes, la primera ronda se basaba en las calificaciones obtenidas en la escuela secundaria y en otros factores.

- Verificación de conocimientos y capacidad académica general:

- Las pruebas evalúan la capacidad para procesar datos, razonar y resumir información.

- Los postulantes deben estar en el $20 \%$ superior de su promoción.

- Verificación de aptitud para ejercer la docencia: Las entrevistas evalúan la motivación para enseñar y aprender, las habilidades de comunicación y la inteligencia emocional.

- Verificación de aptitud para ejercer la docencia: Ejercicios grupales y pruebas prácticas para evaluar habilidades interpersonales y de comunicación.

- Una vez completada la capacitación docente, los candidatos son reclutados por las escuelas individualmente.
Apenas 1 de cada 6

postulantes es

aceptado como docente

\section{Apenas 1 de cada 10 postulantes es aceptado como docente*}


Continúa en otras instituciones formadoras como: "Las universidades que, a través de facultades de educación u otras unidades académicas, forman docentes en algunos países para todo el sistema escolar y en otros exclusivamente para la educación secundaria. Estas instituciones realizan también investigación y extensión educativa". Las Universidades Pedagógicas, creadas "como una estrategia para mejorar la formación inicial y permanente de los docentes, fortalecer la investigación académica como un medio para resolver problemas educativos y servir a la vez como referente cultural en las sociedades donde se desempeñan" (p.39), tal es el caso de la Universidad Pedagógica Nacional de Colombia. El tiempo de duración de los estudios en las escuelas normales colombianas es de dos años, mientras que las licenciaturas universitarias exigen cinco años de estudios.

En el mismo documento se expresa que hay debilidades en la formación docente relacionadas con:

Desarrollo de habilidades y actitudes pertinentes para la práctica profesional; formación en ámbitos como valores ciudadanos; conocimiento de lenguas extranjeras; formación para las competencias del mundo global y la apropiación y uso pedagógico de TIC y, en general, la escasa articulación con las reformas curriculares. (p.44)

¿Qué significado social tiene la profesión docente?, el documento menciona que "el estatus disminuido de la profesión docente en comparación con otras carreras universitarias genera una presión familiar y social hacia los jóvenes interesados en la enseñanza para que busquen otras alternativas más promisorias desde el punto de vista económico y de prestigio social" (p.49). En este sentido, no resulta atractiva la elección de la docencia como una profesión. Buscando que ingresen mejores candidatos a la formación, algunos sistemas han tomado acciones, como es el caso del Perú, que ha elevado el puntaje mínimo requerido en el examen de ingreso, México aplica un examen al inicio del proceso de formación docente.

El estudio efectuado por la UNESCO (2012) determina aspectos críticos sobre la formación inicial y continuada, los cuales en términos generales se enuncian en la tabla 3.

Tabla 3. Aspectos críticos en la formación docente

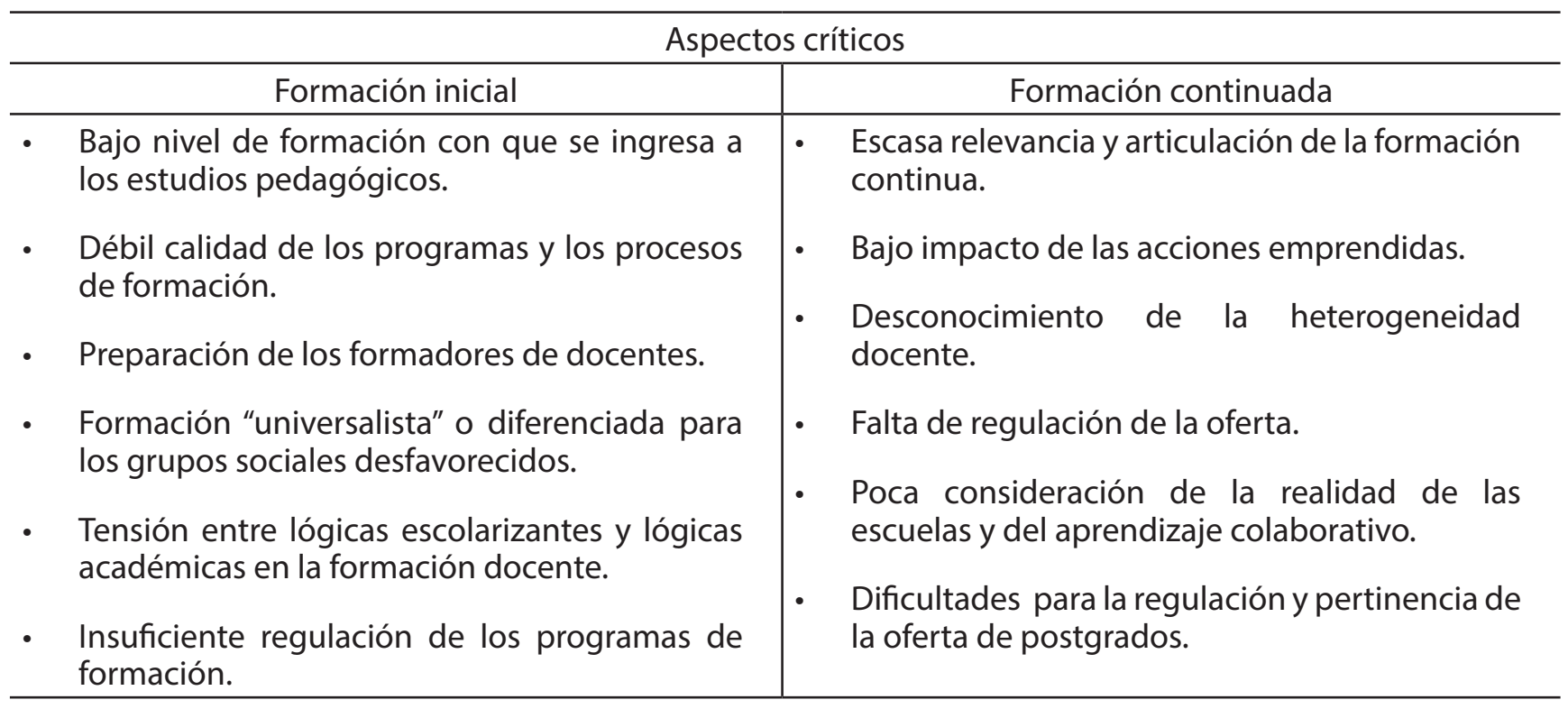

Fuente: Elaboración propia según información de los Antecedentes y criterios para la elaboración de políticas docentes en América Latina y el Caribe. UNESCO, 2012. 
La información anterior si bien se presenta en este artículo en términos generales, si constituye un referente de análisis para las instituciones comprometidas con la formación de docentes, además considerar del documento referente de la UNESCO, algunas orientaciones o criterios para tratar en las políticas docentes, los cuáles remiten a cuatro dimensiones: Priorizar las políticas docentes en una perspectiva sistémica; lograr mayor efectividad de las políticas docentes conciliando criterios de continuidad y cambio; promover la participación de los actores en la generación de políticas y fortalecer la institucionalidad pública para el desarrollo de las políticas docentes (UNESCO, 2012, p.149).

En el contexto nacional y haciendo referencia a los cambios generados en el sistema educativo en los últimos 15 años, el documento del MEN (2012) Políticas y sistema colombiano de formación y desarrollo profesional docente, indica que el Plan Decenal 1999-2005 determina la conformación del Sistema Nacional de Formación de Educadores, desde el cual se trazan los siguientes propósitos:

- Asumir la formación y el desarrollo integral del educador como uno de los factores que ejercen mayor influencia en el logro de una educación de calidad y articularlo con las políticas y la dinámica del sistema educativo en su conjunto.

- Crear las bases para la consolidación de una comunidad académica de la pedagogía que impulse la investigación avanzada y cuyos procesos y resultados tengan un influjo efectivo en la transformación de los programas, de formación. En este sentido impulsar la creación de Institutos Superiores de Pedagogía.

- Establecer los requisitos básicos de creación y funcionamiento de instituciones y programas de formación de los educadores y los criterios que permitan su adecuación a las condiciones de la realidad nacional a los avances del conocimiento en general y de la pedagogía en particular y a las políticas y normas vigentes.

- Fortalecer el ejercicio responsable de la autonomía de las instituciones y de las entidades territoriales y armonizarlas con las obligaciones que tiene a nivel el nivel central del estado en estos aspectos. (p.42)

En el marco de la política "Revolución Educativa", el plan sectorial educativo 2002-2006, define tres políticas: cobertura, calidad y eficiencia, se suma a esto en el plan sectorial años 2006-2010, "la necesidad de una educación más pertinente frente a las demandas de un mundo globalizado, como herramienta para construir un país más competitivo, el desarrollo profesional de los docentes" (p.51). El Plan Decenal de Educación 2006 - 2015, respecto a la formación de docentes, define la necesidad de un sistema de formación, que articule los distintos niveles y núcleos de formación, la coordinación de planes entre las instituciones formadoras, los centros educativos y las instancias de la dirección educativa, a nivel nacional, regional y local.

¿Cómo hacer efectiva la anterior normatividad y hacer de la docencia una profesión?, una posible vía seria potenciar la formación en investigación, subrayando lo expresado por Mckernan (1999) “No es posible ser un profesor - profesional sin comprometerse en la investigación para mejorar el propio rendimiento" (p.67). La investigación perfecciona la enseñanza, dice Rudduck \& Hopkins (2004) al referir a Stenhouse, porque ofrece hipótesis posibles de comprobar en el aula (p.13), agregan además que, los profesores se encuentran a cargo de las aulas y estas constituyen espacios para la comprobación de teorías educativas, "el profesor es un observador participante potencial en las aulas y las escuelas" (p.37).

La investigación en el ámbito educativo ¿De qué investigación se habla al relacionarla con la calidad de los docentes?

La investigación en términos globales comprende dos tipos: básica y aplicada. De la in- 
vestigación básica, Rodríguez Moguel (2005) dice que "busca el progreso científico y su importancia reside en que presenta amplias generalizaciones y niveles de abstracciones con miras a formulaciones hipotéticas de posible aplicación posterior", (p.23), la finalidad de la investigación básica en términos de McDaniel $\&$ Gates (2005) radica en que "trata de ampliar las fronteras del conocimiento; no está orientada a un problema pragmático especifico" (p.10). La perspectiva de Tamayo (2004) señala que "se apoya dentro de un contexto histórico y su propósito fundamental es el desarrollar teoría mediante el descubrimiento de amplias generalizaciones o principios" (p.43).

La investigación aplicada constituye otro tipo de investigación, es una investigación de intervención o de acción sobre problemas específicos con fines de mejoramiento, cambio o transformación de los procesos de enseñanza y aprendizaje; Giroux \& Tremblay (2004) consideran que "lo propio de la investigación aplicada es estudiar problemas concretos con objeto de proponer un plan de acción, para decidir o intervenir eficazmente en una situación dada", (p.38); en palabras de Tamayo (1999) "es un estudio y aplicación de la investigación a problemas concretos, en circunstancias y características concretas [...] se dirige a su aplicación inmediata y no al desarrollo de teorías" (p.51).

Las conceptualizaciones expuestas muestran que hay diferencias sustanciales entre la investigación aplicada y la investigación básica, sin embargo hay una relación de complemento como lo expone Martínez González (2007):

Una y otra se complementan mutuamente: la práctica educativa necesita de las teorías, reflexiones y métodos que se van generando a través de la investigación básica para identificar, entre otras cosas, sus aspectos más positivos y sus limitaciones, para conocer mejor los efectos a los que dicha práctica da lugar, para mejorarlos y para introducir innovaciones progresivas que, siendo es- tudiadas y analizadas por la investigación aplicada, permitan alcanzar objetivos educativos cada vez más altos y complejos. Por su parte, la investigación básica necesita de la práctica educativa para no quedarse sólo en reflexiones teóricas acerca del funcionamiento de la realidad y en el planteamiento de hipótesis que no lleguen a probarse en marcos contextuales concretos. Uno y otro tipo de investigación en interacción se enriquecen mutuamente y permiten alcanzar un mejor y mayor conocimiento de la realidad educativa (p.18).

La finalidad de la investigación aplicada es la de tratar situaciones concretas en contexto reales con población activa, en este sentido, el enfoque investigativo pertinente en los procesos de enseñanza y aprendizaje que configura y cualifica la práctica del docente, es el de la investigación - acción, como lo subrayan Zeichner \& Pereira (2006) al considerar la investigación acción como "una investigación sistemática hecha por profesionales sobre sus propias prácticas"(p.70). La investigación - acción busca que el docente sea un crítico de su propia práctica, y haga de la reflexión un proceso continuo y evaluativo, con la intencionalidad de cualificar la enseñanza y el aprendizaje, generar cambios significativos en las prácticas de aula como contexto situado de aprendizaje; se espera que, con la investigación - acción, los docentes y estudiantes como actores protagónicos de la dialéctica entre la enseñanza y el aprendizaje, asuman críticamente su responsabilidad con el cambio y la transformación de la escuela como sistema social y cultural. Epistemológicamente la investigación - acción se sitúa en el paradigma interpretativo y crítico; esto significa en palabras de Carr que:

Desde la perspectiva crítica, la investigación - acción no se limita al uso de técnicas, ni al seguimiento de procedimientos; la formación de los docentes investigadores no puede realizarse sólo en el aula, recibiendo clases. Implica una actitud de búsqueda per- 
manente ante problemas que se enfrentan, un interés por la transformación de las situaciones que se viven en las escuelas en el sentido de la emancipación, una praxis que, como práctica situada y reflexiva, implica el examen crítico del quehacer docente desde una mirada personal, un compromiso con la tarea desempeñada y el establecimiento de fines acordes con el contexto educativo. (Méndez Pardo \& Méndez, 2007, p. 25)

Uno de los antecedentes de la investigaciónacción remite al movimiento del profesor - investigador, en el Reino Unido, bajo el liderazgo de L. Stenhouse al realizar un estudio sistemático sobre el trabajo de otros profesores y siguiendo procedimientos de la investigación en el aula, en palabras de Elliott (2010) "la investigación tuvo que adoptar una postura autocrítica y mostrar buena disposición para someter el propio trabajo al escrutinio de otros" (p.226). Como asegura Elliott (1993) al decir que:

La investigación - acción integra enseñanza y desarrollo del profesor, desarrollo del curriculum y evaluación, investigación y reflexión filosófica en una concepción unificada de práctica reflexiva educativa. Esta concepción unificada tiene consecuencias de poder en la medida en que rechaza una división del trabajo rígida en donde las tareas y roles especializados se distribuyen en actividades organizadas desde un punto de vista jerárquico. (p.73).

Los cambios procedimentales en la práctica de los docentes, significan cambios de paradigma conceptuales y actitudinales, también cambios de orden administrativo en la escuela, puesto que, la investigación -acción, como otras formas de investigar advierten la necesidad del factor tiempo y la reorganización de nuevos roles o formas de interacción entre docentes, estudiantes y directivos, asumir con profesionalismo la mirada crítica del otro, como un espejo que proporciona una lectura objetiva de las acciones, desde fuera, aporta información sujeta a la reflexión, desde el interior del docente que hace investigación, lo cual le representa en la práctica profesionalismo, seguridad, autonomía y reconocimiento, como bien lo expresan Zeichner \& Diniz (2007), al referenciar desde otras voces teóricas y de acuerdo con análisis testimoniales de docentes investigadores sobre su propias prácticas y de la participación en proyectos determinados por la investigación-acción, afirman que:

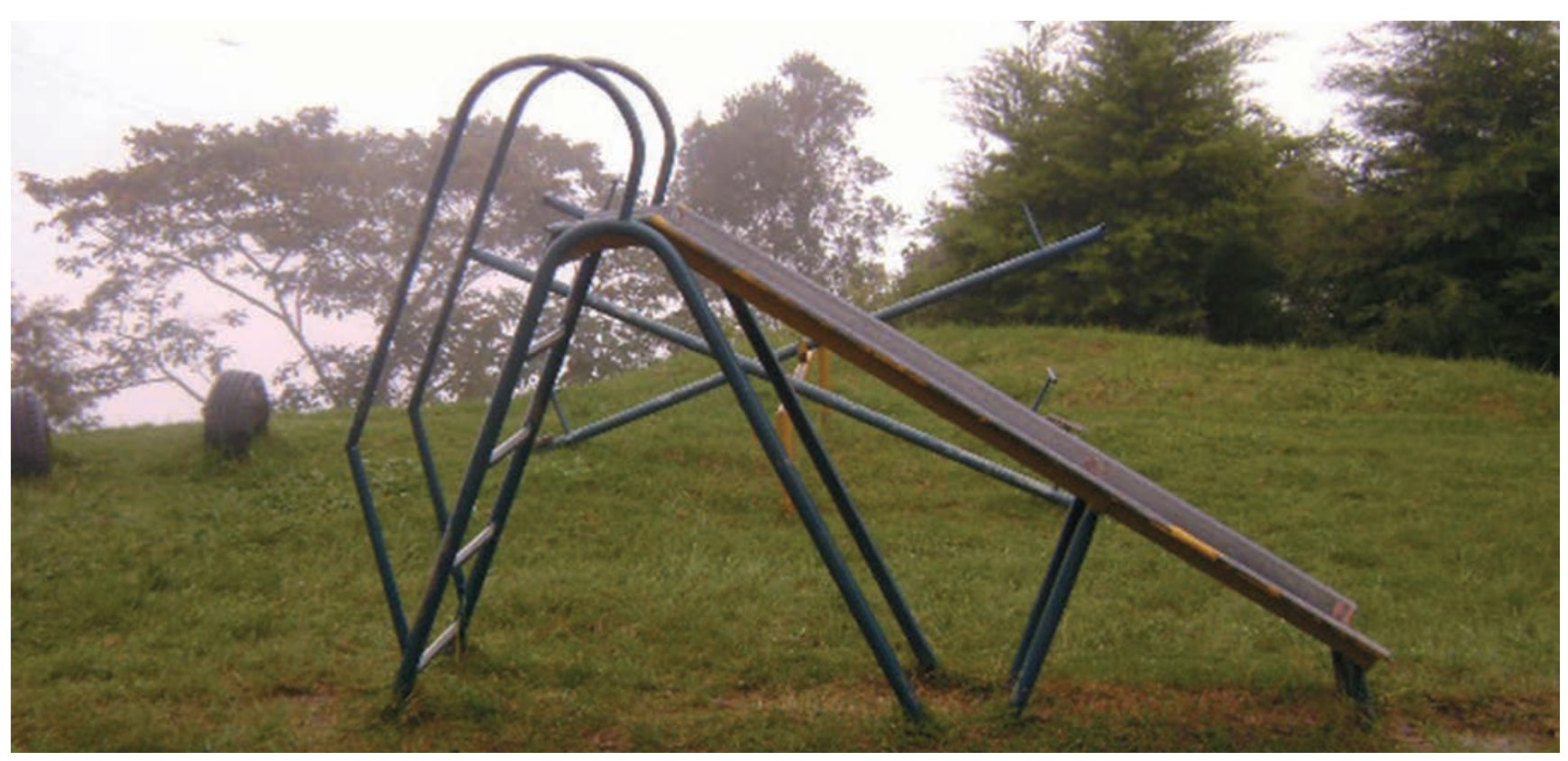


La investigación-acción ha ayudado a un aumento de la confianza y de la autoestima de los profesionales (Webb, 1990; Dadds, 1995; Loucks, et al., 1998); les ayudó a disminuir las distancias entre las aspiraciones y las realizaciones en la profesión (Elliot, Adelman, 1993; Elliot, 1980); ha sido un instrumento importante para que los profesores entiendan de modo más profundo y crítico su propia práctica y para repasar sus teorías personales de enseñanza (Kemmis, 1985), haciéndolos más abiertos y receptivos a las nuevas ideas, más independientes en relación a la autoridad externa (Holly, 1990, p.73).

Aunque desde la teoría se muestren los aciertos de la investigación - acción en el cambio de las prácticas de los docentes y sus efectos en la organización y perspectiva del currículo, solo se hace dinámica y experiencial una vez se constituya en una cultura de cualificación de la práctica docente, bien sea en la formación de docentes noveles, o en el transcurso de docentes con experticia en la profesión.

La investigación en la cualificación de la práctica docente ¿Qué se hace desde la Escuela de Educación de la Universidad Industrial de Santander?

A nivel del pregrado, la Escuela de Educación de la Universidad Industrial de Santander, ofrece dos programas de licenciatura en educación básica, énfasis en lengua castellana, y ciencias naturales y educación ambiental, el currículo de estos programas incluye formación en los campos: disciplinar, pedagógico, básico, investigación y práctica docente. El campo de la investigación aproxima a los estudiantes a la investigación cualitativa y cuantitativa, sus diferenciaciones conceptuales y metodológicas; el campo de la práctica docente, es el espacio curricular, en el que los docentes en formación se acercan a las instituciones educativas, aquí manifiestan las competencias en las que se están formado y encuentran la necesidad de otras en el escenario real para el ejercicio de la docencia. El estudiante practicante se enfrenta a una realidad escolar y a docentes con larga o corta trayectoria que les da experiencia en el ejercicio de la docencia, situarlos en el contexto escolar marca una relación entre docentes expertos y docentes noveles. Los cambios que plantea la actualidad direccionada por la tecnología y la economía global, inciden directamente en la escuela y en la necesidad de cambio en la formación de docentes, como bien lo señala Pérez Gómez (2010) al decir de los docentes en formación:

Han de vivir desde la etapa de formación inicial la complejidad, la incertidumbre y la tensión de la vida real del aula, del centro escolar y de la comunidad educativa arropados por una efectiva y activa cooperación entre iguales y tutorizados, cuando fuere necesario, por profesionales expertos que acompañan y orientan su formación y su actuación, provocando la reflexión sobre la situación, sobre ellos mismos, sobre su actuación, y sobre las consecuencias de la acción. (p.19)

La práctica docente como espacio de formación curricular define la investigación acción, incorporada estratégica y metodológicamente al proyecto de aula, teniendo en cuenta que, al realizarlo rigurosamente con fines investigativos, rompe el paradigma de la enseñanza tradicional. Desde la mirada de W. Kilpatrick (1918), el proyecto de aula es "una propuesta entusiasta de acción para desarrollar en un ambiente social" (Torres, 2006, p.201). A través del método de proyectos, Kilpatrick encuentra la forma de hacer que, los estudiantes se involucren y comprometan con las cosas que le encuentran sentido, "el método de los proyectos nos da un ejemplo de la continuidad de la "educación" y la "vida", del saber y el hacer", (Landon, 2000, p.9); "un buen proyecto curricular tiene que resultar placentero y educativo a la vez; tiene que facilitar una cierta continuidad de los aprendizajes..." (Torres, 2006, p.204). 
El proyecto de aula como estrategia de investigación, posibilita la integración de diversos campos del saber a la vez que potencia diferentes procesos de pensamiento, así el aprendizaje tiene sentido en la medida que es aplicable; como estrategia de investigación en el aula, posibilita la articulación de dos tipos de conocimiento, en términos de Pozo (2006), el conocimiento declarativo, o conceptual, verbalizable, saber qué es, el conocimiento procedimental, saber cómo hacer, cómo transferir o traducir el conocimiento declarativo en acción, estos conocimientos constituyen la base de la interrelación teórico práctica.

El proyecto de aula favorece la interrelación de distintos campos de conocimiento, es una opción para plantear relaciones interdisciplinares, promueve el trabajo en equipo y la contextualización de problemáticas sociales, ambientales o culturales de interés para los estudiantes, además promueve actitud y acciones investigativas tanto en docentes como estudiantes, buscando con esto introducir una mirada investigativa al proceso de enseñanza y aprendizaje, aproximar a los estudiantes practicantes a la necesidad reflexiva de la práctica, considerarla como un proceso investigativo, que bien los sitúan en la ruta de la investigación - acción, buscando conseguir con esto; en palabras de Elliott, (1993) que

(...)la investigación - acción perfeccione la práctica mediante el desarrollo de las capacidades de discriminación y de juicio del profesional en situaciones concretas, complejas y humanas. Unifica la investigación, el perfeccionamiento de la práctica y el desarrollo de las personas en su ejercicio profesional (p. 70).

La formación de los nuevos docentes ha de posicionar la investigación en el contexto escolar y en el escenario más directo de la práctica, el aula, como una de las posibilidades de cambio en la escuela, de empoderamiento de la profesión docente y de transformación e innovación en la práctica, ¿cómo lograrlo?
Las reformas curriculares en un ámbito social surgen cuando el currículo establecido no responde a los propósitos planteados, o bien cuando hay cambios en la estructura social y esto plantea la necesidad de nuevo modelo educativo, en consecuencia pedagógico, curricular y didáctico; una reforma curricular es el producto de un análisis crítico y evaluativo sobre procesos, procedimientos, conocimientos, necesidades, problemáticas y efectividad de los objetivos de formación en la escuela de cara a la sociedad. El movimiento de reforma curricular, que surge en Inglaterra hacia los años sesenta crea el movimiento de los profesores como investigadores, en el que participa activamente J. Elliott (1993), y de esta experiencia señala que :

Las prácticas curriculares no se derivaban de teorías del curriculum elaboradas y comprobadas con independencia de esas prácticas. Constituían el medio a través del cual elaboramos y comprobamos nuestras propias teorías y las de los demás [...] recogimos datos empíricos sobre sus efectos, utilizándolos para fundamentar nuestras elaboraciones teóricas desarrolladas en colaboración en un contexto de responsabilidad colegiada. No lo denominamos investigación y mucho menos investigación - acción. Esta organización vino mucho después, cuando el mundo universitario respondió al cambio en las escuelas (p.21)

Metodológicamente la práctica docente en los programas de licenciatura, se desarrolla siguiendo las etapas propias de la investigación acción, y se definen así:

Primera etapa. Diagnóstico. Momento inicial del proyecto en el que se sitúa al estudiante de práctica docente en una institución educativa, allí explora, reconoce y caracteriza el entorno educativo. Por medio de un diagnóstico formal el estudiante de práctica docente podrá determinar las fortalezas y debilidades de los estudiantes, los vacíos de los progra- 
mas del currículo, y todo aquello que a través de proyectos alternativos pueda ayudar a resolver (Cerda, 2008, p.63). La fase de diagnóstico se apoya en técnicas de investigación cualitativa como la observación, los estudiantes siguen el diario de campo, y una guía de trabajo que orienta el diagnóstico institucional y el diagnóstico de aula. En esta fase y según análisis de la información interpretan intereses, necesidades y preguntas de los estudiantes, finalmente se determina una situación problémica, de acuerdo con esta, se definen objetivos y proceso metodológico para dar tratamiento a la situación.

Segunda etapa. Planificación del proyecto. De acuerdo con el problema definido, se traza el Plan de Acción, esto implica tomar decisiones respecto a: cuáles estándares curriculares básicos de competencia y qué procesos de pensamiento se desarrollarán, qué estrategias didácticas y actividades de enseñanza y aprendizaje son necesarias para dar solución al problema, qué recursos, tiempos y espacios de aprendizaje son determinantes en el tratamiento del problema, además, especificar cómo evaluar el plan de acción y cada una las actividades de enseñanza y aprendizaje propuestas. La estructuración del proyecto de aula, bien la define Cerda (2008) al expresar que, dar curso a la planificación del proyecto de aula, el docente puede plantearse objetivos con frecuencias de tiempo cortas, diarias, semanales o mensuales, plantear unidades de trabajo a partir de ciertas expectativas de logros, reorganizar contenidos de acuerdo con los criterios a adoptar, por ejemplo, asignaciones de tiempo, selección, organización y secuenciación de actividades de aprendizaje y discusión de recursos didácticos (p.86).

Tercera etapa. Ejecución o desarrollo del proyecto. Puesta en marcha del proyecto y consecución de los propósitos y del plan de acción. La realización del proyecto de aula está guiada por los objetivos planteados, la problemática definida y el plan de acción propuesto. Se sugiere a los docentes asesores y estudiantes de práctica docente seguir instrumentos como el diario de clase y protocolos de trabajo individual y grupal, en los que se registra la información que surge de acuerdo al desarrollo del plan de acción, esta información es el sustrato o base objeto de reflexión y replanteamiento de las acciones.

Cuarta fase. Evaluación del proyecto. La evaluación sigue tres instancias: Autoevaluación, el estudiante practicante evalúa su proceso, se genera una relación de dialogo crítico entre el docente asesor de práctica y el estudiante practicante; heteroevaluación, la efectúan el docente titular de la institución educativa y el docente asesor de práctica; Co-evaluación, se da entre el estudiante practicante y el grupo de estudiantes participes en el proyecto de aula. La evaluación se efectúa teniendo en cuenta instrumentos y criterios definidos para cada instancia. Es de subrayar que las tres miradas evaluativas sobre el proceso y el desempeño de los estudiantes practicantes, proporcionan una lectura global que permite analizar los niveles de logro entre los objetivos propuestos y los resultados alcanzados, valorar alcances de las acciones, analizar las alternativas de solución propuestas, la efectividad de las estrategias, actividades y situaciones de aprendizaje, los problemas que surgen, los resultados finales del proyecto y el desempeño profesional de los estudiantes practicantes.

Finalmente, los resultados del proyecto de aula se socializan en las instituciones educativas donde se efectúa la práctica docente y en el colectivo o grupo de estudiantes practicantes; a partir de esta experiencia los estudiantes practicantes encuentran intereses investigativos que logran abordar como trabajo de investigación, o trabajo de grado, requisito necesario para titularse como licenciado en educación básica.

Definir el proyecto de aula, y a través de éste desarrollar un proceso de investigación - acción, permite aproximar y dar participación activa a los estudiantes de la educación básica en 
procedimiento investigativos; establece bases de formación en investigación en el aula en los estudiantes de licenciatura, docentes en formación; genera una cultura de sistematización y análisis de procesos con fines investigativos. También permite situar a los futuros profesionales de la educación en contextos reales laborales, que funcionan con el marco de políticas curriculares, orientadas al desarrollo de competencias, lo cual demanda la integración de conocimientos y de procesos de enseñanza y aprendizaje entre la educación básica y la educación superior, y de creación de vínculos académicos entre las instituciones educativas y la universidad.

A nivel de Posgrado la Escuela de Educación ofrece el programa de Maestría en Pedagogía, con seis líneas de investigación:

- Concepciones y prácticas pedagógicas

- Formación ciudadana: democracia y convivencia escolar,

- Lenguaje y pedagogía

- Tecnologías de la información y la comunicación aplicadas a la educación

- Calidad educativa y gestión escolar

- Construcción del saber pedagógico y didáctico de las ciencias naturales y sociales.

Durante los veinte años del programa de Maestría, la línea de investigación Construcción del saber pedagógico y didáctico en ciencias naturales, se configura como un escenario de análisis, interpretación y problematización de los procesos de enseñanza y aprendizaje, objeto de estudio de la didáctica. La finalidad de la línea es la de cualificar la formación profesional de los docentes de ciencias naturales y sociales considerando la didáctica y los aspectos que la comprenden como una necesidad investigativa manifiesta en las prácticas docentes.

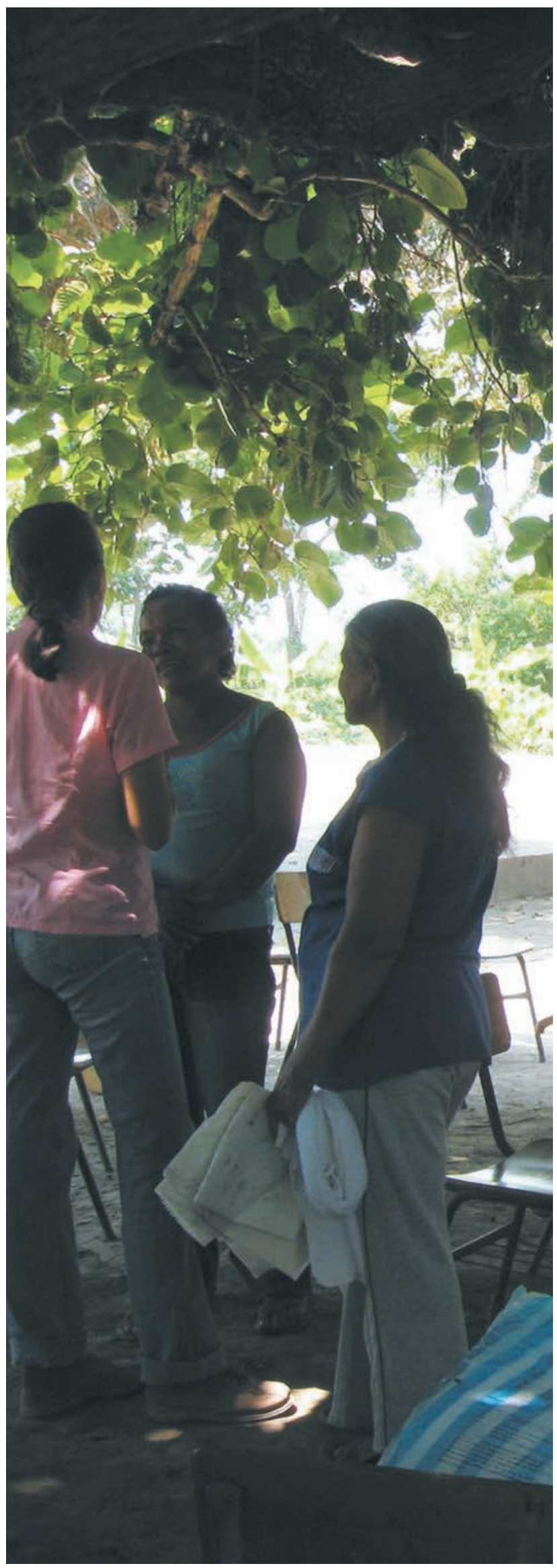




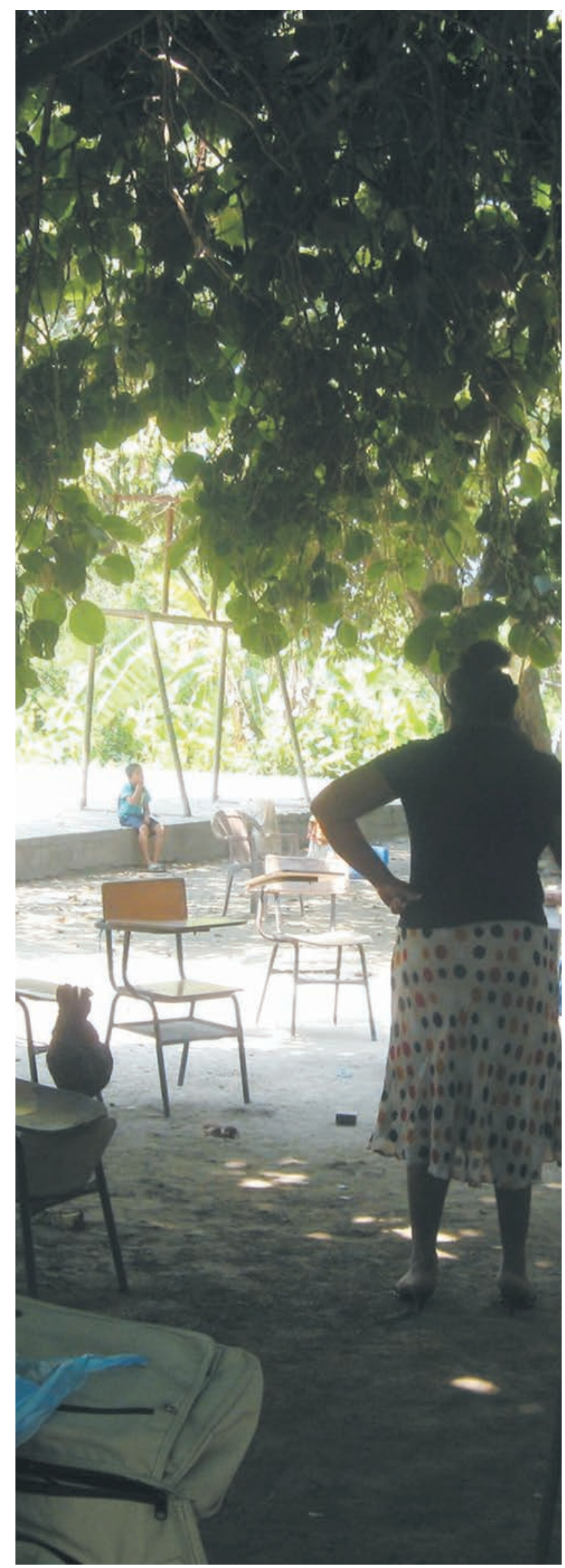

Los procesos de investigación en esta línea, constituyen tres momentos, el primero hacia el año de 1994 conformado por un grupo de docentes de los programas de Especialización en Docencia Universitaria y de Maestría en Pedagogía, quienes plantean la necesidad de abordar problemas de carácter didáctico y generar cuestionamientos en torno a las concepciones y prácticas tradicionales; la finalidad de este grupo es la de generar modelos pedagógicos sustentados en la investigación. Un segundo momento, el del año 2004, durante el cual se estructura y da sustento teórico a la línea de investigación y define como los propósitos de acción los siguientes:

- Cualificación e innovación de los procesos de enseñanza y aprendizaje de la ciencia.

- Constitución de una cultura escolar y científica en los niveles de educación básica, media y superior.

- Generación de conocimiento didáctico en el campo de la ciencia.

- Investigación de situaciones y problemáticas que surgen en los procesos de enseñanza y aprendizaje de la ciencia.

Los pilares de investigación de la línea se determinan por la interacción entre docentes y estudiantes en contextos reales y naturales, esto es, las aulas de clase y lo que aquí sucede, los procesos de enseñanza y aprendizaje, objeto de estudio de la didáctica. Durante este momento y hasta el año 2012, se inscriben y desarrollan seis proyectos de investigación, caracterizados por definir la "investigación aplicada", el método de investigación acción (cinco proyectos) y la investigación cuasi experimental (un proyecto). La investigación - acción, es investigación de intervención directa sobre el problema planteado, incide directamente en el currículo escolar y en la profesionalización de los docentes, si se asume como parte constitutiva de la práctica docente. 
La pregunta investigativa y clave se orienta al cuestionamiento - cómo-, considerado interrogante de indagación procedimental cognitiva, el tratamiento de éste incluye, estrategias de tipo didáctico que buscan mejorar aprendizajes de objetos disciplinares específicos, por ejemplo, el concepto de integral, ecosistema, tabla periódica, fracción, además desarrollar procesos de pensamiento científico, y en consecuencia, competencias científicas y comunicativas. Las preguntas investigativas que se plantean los estudiantes de la maestría, autores de los trabajos de investigación, se caracterizan por ser claras, concretas, pertinentes y necesarias de resolver según problemática actual de la realidad escolar. Son preguntas propias de la investigación cualitativa, de acuerdo con Connolly (1998), o también como afirman Abreu, Parra González, \& Molina Arenas (2012) "tratan de obtener conocimientos sobre determinados procesos educativos, familiares y sociales y las experiencias que existen en una ubicación específica y el contexto" (p.184).

El problema de investigación se plantea en instituciones educativas de carácter privado (dos) y de carácter oficial (4), en los niveles de educación superior, (dos), básica secundaria (dos) y básica primaria (dos); el desarrollo de los proyectos de investigación ha generado hasta ahora, la participación de 58 estudiantes de educación superior, 52 de educación básica secundaria y 45 de la educación básica primaria, el total de la población escolar participante en el segundo momento de la maestría es de 155 estudiantes de los tres niveles de educación. Los anteriores datos solo son significativos para la línea de investigación, sin embargo muestran la atención que se da a los niveles de escolaridad y la vinculación activa de estudiantes de la educación básica, media o superior, y de docentes/autores de cada uno de los proyectos como participantes activos en el proceso de investigación. Se definen técnicas e instrumentos de investigación pertinentes a la investigación acción, como la observación participante, en- trevista estructurada, encuesta, talleres de investigación, pruebas de pretest y postest, la información que se indaga, se registra siguiendo el diario de clase o diario de campo, cuestionarios, cuadernos de los estudiantes, protocolos de encuesta, entrevista, guía de observación y unidades didácticas. El diseño metodológico se estructura en fases y/o etapas de investigación, que incluyen: fundamentación teórica y problematización, identificación y análisis del problema, indagación de conocimiento previo, análisis de información, diseño e implementación, de la estrategia o del modelo didáctico, aplicación y seguimiento, análisis e interpretación de resultados y replanteamiento de la acción.

El tercer momento, surge en el año 2011, marcado por definir el trabajo de investigación bajo la modalidad de macroproyecto, se da la integración entre las líneas de investigación: lenguaje y comunicación, TIC aplicadas a la educación y la línea construcción del saber pedagógico y didáctico de las ciencias naturales, se plantea el macroproyecto "El texto electrónico en el desarrollo de competencias científicas y comunicativas". En el marco de este macroproyecto se realizaron tres investigaciones en contextos escolares de la educación básica, sector urbano oficial y privado, y oficial rural. Una de estas investigaciones implementa el programa de filosofía para niños FpN-como propuesta para promover el desarroIlo de competencias científicas y comunicativas con la mediación de TIC. Caso: estudiantes de séptimo grado de una institución educativa oficial de Bucaramanga; el desarrollo del programa de filosofía para niños implementa como mediación los textos electrónicos a través del edublog y del eduwiki. Una segunda investigación define El proyecto Noria en el desarrollo de competencias científicas y comunicativas y la incorporación del uso del blog: caso estudiantes de cuarto grado de educación básica de un colegio privado de Girón. Un tercer trabajo de investigación que se encuentra en curso, 
define El proyecto de aula investigativo como estrategia didáctica para la formación de competencias científicas y comunicativas. Caso: colegio público-rural de Puerto Parra - Santander'; trabajo que a la fecha prepara el informe final, ha sido inscrito por su autor al premio Compartir al maestro 2013-2014, será visitado por los evaluadores de la Fundación Compartir².

Los anteriores trabajos evidencian que sí es posible replantear la práctica docente, sustituir un modelo de enseñanza tradicional, por un modelo investigativo, dejar de lado estrategias didácticas discursivas que limitan la participación activa de los estudiantes, apoyar las acciones de estudiantes y docentes utilizando como mediación la tecnología, sin embargo lograr, el desarrollo de competencias, es una tarea constante, no solo del docente autor de trabajos de investigación, sino otros colegas que interactúan con los escolares desde otros campos del conocimiento.

\section{Conclusión}

¡El desarrollo de un país depende de la educación de su sociedad!, premisa que compromete a cada una de las partes del sistema educativo, trabajar en concordancia por la cualificación y profesionalización en los procesos de formación de escolares y docentes. La realidad actual plantea una nueva escuela, un nuevo docente caracterizado además por ser "personalmente responsable de la resolución de problemas en un mundo que es personalmente inestable", señala Popkewitz (1997, p 56); el presente nos reclama un cambio de paradigma, de pensamiento y de acciones que confluyan en la generación de conocimientos y de seres humanos, humanizados.

Indicadores de las pruebas censales, constituyen un diagnóstico de la calidad de la educación, estas pruebas en el orden nacional se vienen aplicando desde el 2002; a partir del 2012 la prueba Saber es anual para los nive1 Rodríguez Baños J.

2 http://www.premiocompartir.org/maestro/ les de tercer grado, a quienes se les evalúa competencias en lenguaje y matemáticas, considerados conocimientos básicos; al nivel de quinto grado de básica primaria y noveno grado de básica secundaria, se les evalúa competencias en lenguaje, matemáticas, ciencias naturales y competencias ciudadanas. Los resultados logrados desde el inicio de la aplicación de las pruebas, al igual que los resultados internacionales, caso PISA de 2006 y 2009, han de constituirse en el sustrato de indagación investigativa que aporte soluciones al mejoramiento de procesos de pensamiento en los escolares, los cuales manifiesta en las competencias anteriormente mencionadas. Utilizar los resultados para efectuar evaluación de otros aspectos que inciden en mejores aprendizajes, como son, la práctica pedagógica de los docentes, el currículo institucional y de las áreas de formación, las formas de evaluación, la distribución del tiempo escolar, entre otros.

El posicionar la investigación en la formación docente significa asumirla como una impronta que caracterizaría los programas académicos en las Instituciones Educativas que los brindan, en consecuencia, replantearía la concepción y organización del currículo, y el punto de partida, o mejor el horizonte de formación, el modelo pedagógico. La realidad es compleja, es de cambios vertiginosos, con una dinámica más de incertidumbres que de certezas, hecho que nos expone a otro tipo de racionalidad, conducente a crear una escuela para hoy, artífice del mañana.

La cualificación y los cambios sustanciales en la práctica docente, se construyen en la medida que se participa de programas de formación articulados en lo académico e investigativo, brindan al docente un medio para generar una cultura crítica y reflexiva sobre la propia práctica. Posicionar en el aula de clase la investigación - acción, exige, pero también construye otro tipo de roles, acciones y valores, configurados en una comunidad de 


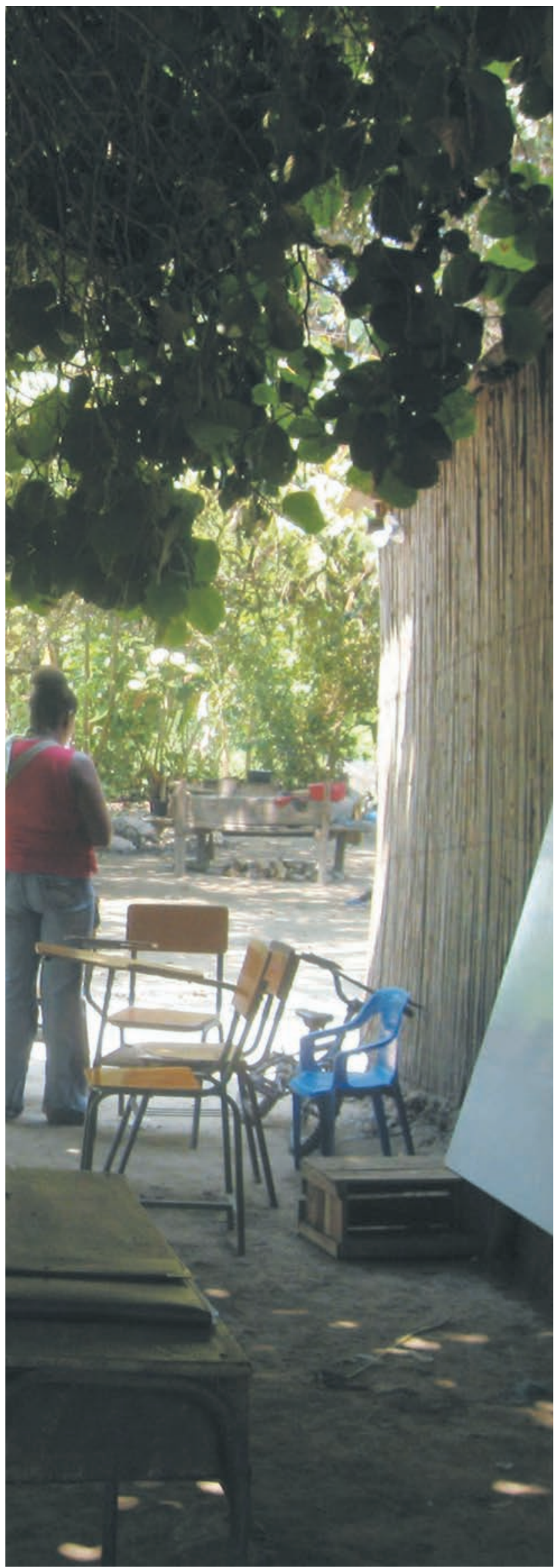

indagación, que problematiza y propone, en tanto es investigativa. Sin embargo esto exige al sistema educativo, brindar al docente incentivos para que pueda acceder a programas de formación, en el nivel de posgrado; exige al docente compromiso y profesionalismo, representado en la trascendencia o el impacto que pueda tener los programas de cualificación en el ámbito escolar y en mejores aprendizajes.

\section{Referencias}

Barber, M. \& Mourshed, M. (2008). Cómo hicieron los sistemas educativos con mejor desempeño del mundo para alcanzar sus objetivos. Chile. Programa de Promoción de la Reforma Educativa en América Latina y el Caribe. Disponible en: http://www.oei.es/pdfs/documento_preal41.pdf

Cerda, H. (2008). El proyecto de aula. Bogotá, Colombia: Magisterio.

Elliott, J. (1993). El cambio educativo desde la investigación acción. ( $3^{\mathrm{a}}$ ed.) Madrid: Morata

Elliott, J. (2010). El "estudio de la enseñanza y del aprendizaje": una forma globalizadora de investigación del profesorado. Revista Interuniversitaria de Formación del Profesorado, 68 (24,2), 223-242. Disponible en: http://www.aufop.com/aufop/uploaded_files/articulos/1279268560.pdf

Giroux, S. \& Tremblay, G. (2004). Metodología de las ciencias humanas. México: Fondo económico de cultura.

Martínez González, R.A., (2007) La investigación en la práctica educativa: Guía metodológica de investigación para el diagnóstico y evaluación en los centros docentes. Colección: investigamos № 5 . España: Ministerio de Educación y Ciencia. Disponible en: http://www. gse.upenn.edu/pdf/La\%20investigaci\%C3\%B3n\%20 en\%20la\%20pr\%C3\%A1ctica\%20educativa.pdf

McDaniel, C., \& Gates, R. (2005) Investigación de mercados. México:Thomson.

McKernan, J. (1996). Investigación - Acción y curriculum. Madrid: Morata.

Mejía Páez, L. M. (2013). Tesis de Maestría. Universidad Industrial de Santander, Bucaramanga, Colombia.

Méndez, A., \& Méndez, S. (2007) El docente investigador en educación. Textos de Wilfred Carr. México: Uni- 
versidad de ciencias y artes de Chiapas. Disponible en:http://www.aiu.edu/applications/DocumentLibraryManager/upload/Susy\%20Mendez\%20Pardo. pdf

Ministerio de Educción Nacional. (2012). Políticas y sistema colombiano de formación y desarrollo profesional docente. Disponible en: http://www.colombiaaprende.edu.co/html/productos/1685/articles-312233_ documentobaseop.pdf

Organización para la Cooperación y el Desarrollo Económico (2010). Resultados PISA 2009: Resumen Ejecutivo. Disponible en: http://www.eduteka.org/pdfdir/ Pisa2009.pdf

Organización de las Naciones Unidas para la Educación, la Ciencia y la Cultura. (2012). Antecedentes y Criterios para la Elaboración de Políticas Docentes en América Latina y el Caribe. Chile: UNESCO. Disponible en: http://www.orealc.cl/educacionpost2015/wp-content/blogs.dir/19/files_mf/antecedentesycriteriospar apol\%C3\%ADticaspublicasparadocentesfinal.pdf

Pérez, A. (2010). Nuevas exigencias y escenarios para la profesión docente en la era de la información y de la incertidumbre. Revista Interuniversitaria de Formación del Profesorado, $\mathrm{N}^{\circ}$ 68, 24,2, pp. 17-36. Recuperado en: http://www.aufop.com/aufop/uploaded_files/ congresos/1296516384.pdf
Popkewitz, T. (1997) La profesionalización, el gobierno del profesor y el conocimiento académico: algunas notas comparativas. Revista interuniversitaria de formación del profesorado, 29, 89-109.

Pozo, J. (2006) Aprender a enseñar ciencia. Madrid: Morata.

Rodríguez Moguel, E. (2005) Metodología de la investigación. México: Universidad Juárez Autónoma de Tabasco.

Stenhouse, L. (2004). La investigación como base de la enseñanza. Selección de textos por Rudduck, J. \& Hopkins. Quinta edición. Madrid: Morata.

Tamayo \& Tamayo, M. (1999). El proceso de la investigación científica. México: Limusa.

Torres, J. (2006). Globalización e interdisciplinariedad. EI currículo integrado. Quinta edición. Madrid: Morata.

Wagner, T. (2011). Finlandia: el sistema escolar más asombroso del mundo. Programa de innovación educativa del centro de tecnología del emprendimiento de Harvard. EE-UU. [Archivo de video] Disponible en: http://youtu.be/yRMWcsqnsmY

Zeichner, M. y Diniz, J. (2007). Investigación de los educadores y formación docente orientada a la transformación social. Revista Docencia, No 31, 6881. Disponible en: http://www.revistadocencia.cl/ pdf/20100731211551.pdf 